\title{
Lidil
}

Revue de linguistique et de didactique des langues

$63 \mid 2021$

Littératie numérique et didactique des langues et des cultures

\section{Littératies numériques adolescentes et perspectives d'enseignement : le cas de la fanfiction}

Adolescent Digital Literacy and Teaching Perspectives: The Case of Fanfiction

\section{Magali Brunel}

\section{(Q) OpenEdition}

\section{Journals}

Édition électronique

URL : http://journals.openedition.org/lidil/9189

DOI : 10.4000/lidil.9189

ISSN : 1960-6052

Éditeur

UGA Éditions/Université Grenoble Alpes

Édition imprimée

ISBN : 978-2-37747-283-3

ISSN : $1146-6480$

\section{Référence électronique}

Magali Brunel, «Littératies numériques adolescentes et perspectives d'enseignement : le cas de la

fanfiction », Lidil [En ligne], 63 | 2021, mis en ligne le 30 avril 2021, consulté le 30 avril 2021. URL :

http://journals.openedition.org/lidil/9189; DOl : https://doi.org/10.4000/lidil.9189

Ce document a été généré automatiquement le 30 avril 2021.

(c) Lidil 


\title{
Littératies numériques adolescentes et perspectives d'enseignement : le cas de la fanfiction
}

\author{
Adolescent Digital Literacy and Teaching Perspectives: The Case of Fanfiction
}

\author{
Magali Brunel
}

\section{Introduction}

1 Parmi les pratiques de littératie numérique contemporaine informelles, les fanfictions constituent un genre prisé (Lacelle et coll., 2017) par les publics jeunes. Elles se définissent comme des récits fictionnels écrits par des fans qui prennent appui sur des œuvres préexistantes admirées, littéraires ou non, qui ont souvent remporté un grand succès populaire (Harry Potter, Twilight, ou encore, pour le cinéma, Star Wars). Ces récits empruntent l'univers diégétique de l'œuvre source et se proposent, "à partir d'un certain évènement de l'intrigue originale, de faire bifurquer l'histoire et de créer un monde parallèle, mais aussi concurrent, de celui qu'avaient élaboré les scénaristes ou l'auteur officiel » (François, 2009, p. 166).

2 Plus largement, les fanfictions forment aujourd'hui des espaces numériques lettrés où se conjuguent plusieurs types de productions. On parle alors, suivant Lacelle et coll. (2017), de blogue de fanfiction, pour désigner cet espace de «rencontre entre l'intra et l'extratextuel » (p. 46). Or, le potentiel didactique de ces formes langagières, bien loin des seules préoccupations technologiques, parait prometteur. Barnabé considère ainsi que, dans les pratiques de fanfiction, « apprendre devient un véritable mode de participation, d'appartenance à la communauté" (Barnabé, 2014, p. 8). En effet, certaines pratiques numériques adolescentes constituent des pratiques littéraciées voire littéraires, et en ce sens, s'approchent des objets d'enseignement du français. Pourtant, elles ne peuvent être transposées dans la classe sans modification, tout l'enjeu de l'adaptation consistant à transformer celles-ci en contexte scolaire sans les dénaturer totalement. 
3 C'est ce qui justifie la tentative que nous avons engagée de manière exploratoire, lors de l'année scolaire 2017-2018, d'adapter un blogue de fanfiction au collège dans un projet d'enseignement-apprentissage. Cependant, se pose la question des risques de cette transposition: si l'objet social s'adapte en contexte scolaire, reste-t-il attractif, permet-il notamment encore de déjouer un rapport à l'écrit scolaire négatif ? C'est la question que nous nous proposons d'examiner dans cette étude. Il s'agira tout d'abord de justifier l'arrière-plan épistémologique et théorique qui légitime notre adaptation didactique de fanfiction. Puis nous examinerons comment, dans le cadre de l'expérimentation que nous avons menée dans une classe de $3^{\mathrm{e}}$, nous pouvons analyser l'évolution de la motivation des élèves et de leur rapport à l'écrit.

\section{Cadre théorique et épistémologique}

\subsection{Les enjeux de l'exploitation de la fanfiction en contexte scolaire}

4 Notre projet d'adaptation des pratiques fanfictionnelles s'inscrit en relation avec une conception de l'enseignement de la littérature comme pratique contribuant à la formation de l'identité de la personne et du citoyen. Nous considérons ainsi qu'il doit permettre le développement d'un sujet lecteur, engagé personnellement dans ses lectures (Mazauric et coll., 2011; Massol \& Rannou, 2017) et la formation de communautés lectorales, qui favorisent le développement culturel et social à l'échelle d'un groupe (Diaz, 2015), permettent le débat et la confrontation et développent la cohésion sociale et le partage de valeurs communes (Sauvaire, 2013).

Il se justifie également du fait que de nombreux élèves entretiennent, surtout à partir du collège, un rapport négatif à la littérature, et plus largement à l'écrit. Or, les travaux de Dabène (1991) Barré-De Miniac (2000) ou Lafont-Terranova (2009) ont montré l'importance de ce rapport dans le processus d'apprentissage. Les expériences passées, les conceptions, la mémoire ou les habitudes influencent en effet la façon dont l'élève aborde une tâche en lecture ou en écriture. Ce rapport à l'écrit est particulièrement mis à mal au moment de l'adolescence : les habitudes de loisirs évoluent et se détournent alors de la lecture, comme l'atteste l'enquête « Les jeunes et leur rapport aux livres et à la lecture " (2016) menée par le CNL, le nombre de livres lus par an étant divisé par trois. Cette détérioration du rapport à l'écrit est confirmée par l'enquête sur les pratiques de lecture en contexte scolaire de Renard (2011) qui en donne plusieurs éléments d'explication : à partir du collège commencent à se distendre les liens entre le lecteur scolairement attendu et le lecteur ordinaire, les compétences lectorales, qui relèvent d'une «façon de lire " plus analytique se dissocient des expériences lectorales ordinaires, et les gouts personnels s'écartent du corpus considéré comme porteur de valeur littéraire.

6 Dans ce contexte, la médiatisation par l'écran peut permettre de contourner un rapport à l'écrit difficile. En effet, comme le souligne Miguet (2014), les lecteurs n'ont pas, à l'égard d'un texte sur écran, le sentiment d'une valeur patrimoniale, mais celui de la facilité de manipulation et de la liberté d'usage. De même, les scripteurs perçoivent l'écran comme un espace attractif, fluide, et en pratiquant d'autres gestes, n'ont pas l'impression de réaliser une tâche d'écriture scolaire habituelle. De plus, la proposition d'écrire sur écran des textes qui ne sont pas associés à des textes scolaires est également susceptible de susciter une vision favorable de la tâche proposée. 


\subsection{La fanfiction, une pratique adolescente présentant des parentés avec les genres écrits scolaires}

7 Parmi les différents genres numériques pratiqués par les jeunes, la fanfiction se prête particulièrement, selon nous, à une adaptation dans le cadre d'un projet d'enseignement de la littérature, car elle entretient des liens avec des pratiques plus traditionnelles, y compris scolaires.

Rappelons qu'une fanfiction consiste dans l'écriture d'une version ou d'une production liée à une œuvre source, dont l'auteur est fan. Celui-ci présente, à travers sa fanfiction, une forme d'appropriation personnelle de l'œuvre. Il est ainsi d'abord un lecteur qui admire, mais un lecteur créatif qui devient à son tour auteur et partage son écrit avec une communauté de lecteurs-fans, qui eux aussi peuvent réagir, écrire et commenter l'écrit. Or, Doueihi (2011) le rappelle, la pratique de l'écriture à partir d'une œuvre source s'inscrit dans la continuité de pratiques culturelles lettrées classiques tout en les renouvelant dans un espace spécifique devenu " un lieu de sociabilité sans précédent " (p. 230). En effet, même si elle apparait comme une pratique contemporaine, la fanfiction trouve des parentés avec des pratiques littéraires très anciennes : l'emprunt, le pastiche ou la transposition sont des procédés bien connus, sources d'exercices scolaires traditionnels, que les pratiques d'enseignement courantes actuelles continuent de prolonger et que les textes officiels en vigueur recommandent (MEN, 2015), ainsi que l'a montré Cauterman (2003), dans la typologie des sujets d'écriture d'invention qu'elle propose: les suites de texte, changements de points de vue ou transpositions sont des sujets fréquents $-\mathrm{y}$ compris d'exercices certificatifs -, comme le montre cette consigne d'une épreuve du brevet des collèges :

Sujet d'invention :

«Il faut que je me lève, que je marche, que je parle à quelqu'un... »

Vous imaginerez la suite du récit, en montrant comment l'intervention d'un autre personnage permet au narrateur de sortir de sa situation. Votre texte devra mêler narration, description et dialogue. Votre texte fera au moins deux pages (soit une cinquantaine de lignes). (Sujet du brevet des collèges 2016, à partir de M. Genevoix, Ceux de 14, 1915)

9 Si les préconisations et consignes sont spécifiquement scolaires, la proposition qui consiste à faire bifurquer un épisode rappelle les pratiques fanfictionnelles. Voici pour exemple l'accroche d'une fanfiction écrite à partir de Bilbo le Hobbit de Tolkien ${ }^{1}$.

Son au-delà [Vue 184] Genres : Romance/Action-Aventure/Heroic Fantasy.

[Publiée le 2015-09-20] [Mise à jour : 2016-02-10]

Vous êtes-vous déjà demandé ce qui vous attendait " après »? L'idée de cette petite

histoire m'est venue après m'être questionnée à ce sujet... et lu du Tolkien,

évidemment ! Notamment « Le Silmarillion » et « Le Hobbit ». (fanfic.fr)

Dans des formes bien différentes, les deux propositions d'écriture envisagent un projet narratif similaire, consistant à se demander « ce qui nous attend après » un moment de la diégèse. Les sujets d'écriture des écrits scolaires révèlent ainsi des convergences avec des productions fanfictionnelles.

11 Mais cette convergence peut également présenter un risque (Brunel, 2018b): si, pour les élèves, les propositions de fanfiction sont trop proches des sujets d'écriture qu'ils pratiquent habituellement ${ }^{2}$, si la manière de travailler proposées par les enseignants restent trop influencées par la forme de la "rédaction », alors l'on peut craindre que 
les élèves ne puissent considérer la pratique de fanfiction comme une occasion de rompre avec des pratiques scolaires.

\subsection{Les liens entre pratiques informelles et pratiques scolaires}

12 Notre projet de recherche vise à favoriser un enseignement de la littérature qui rapproche pratiques culturelles numériques informelles et pratiques scolaires. Pour autant, l'adaptation de la fanfiction à l'école ne s'effectue pas de manière transparente sans modification.

\subsubsection{L'appui sur les compétences déjà-là des élèves} élèves acquises dans le cadre d'apprentissages informels en particulier dans leurs activités privées tournées vers Internet et les réseaux (Ipsos-ONL, 2016). Nous nous référons à la définition retenue par le Conseil de l'Union européenne qui caractérise l'apprentissage informel comme "un apprentissage découlant des activités de la vie quotidienne liées au travail à la famille ou aux loisirs qui n'est ni organisé ni structuré en termes d'objectifs, de temps ou de ressources, [et] peut posséder un caractère non intentionnel de la part de l'apprenant » (Commission européenne, 2012, p. 5).

Comme l'a montré Cordier (2015), les adolescents se livrent à de nombreuses discussions et commentaires en ligne sur les produits culturels qu'ils apprécient. Ils exercent aussi des pratiques de lecture et d'écriture privées largement liées au numérique. Il s'agit alors de s'appuyer sur ce déjà-là, dans un projet d'apprentissage et de «tenter de faire des ponts entre les sphères de déploiement de ces pratiques, faire des ponts entre les apprentissages formels et non formels" (ibid., p. 269). Plus largement, l'enseignant peut amener ses élèves à transférer leurs compétences en littératie numérique d'une sphère de pratiques à une autre, et exhorter «les élèves à exploiter les compétences qu'ils mettent en œuvre avec les objets numériques en dehors de l'école» (ibid., p. 283).

\subsubsection{Accueillir les pratiques numériques culturelles privées dans l'enseignement de la littérature}

Plusieurs chercheurs, dont Poyet (2011) ou Cordier (2015), soulignent les écarts entre les pratiques culturelles numériques personnelles des élèves et les produits culturels classiques et précisent que les objets culturels qui façonnent la culture des adolescents entrent en tension avec ceux de la culture scolaire, et constituent parfois même une façon, pour les jeunes, de se démarquer de la culture de leurs parents ou de l'institution.

16 Pour autant, et sans doute pour cela même, Poyet (2011), tout comme Cordier (2015), justifient l'importance du rapprochement entre les pratiques culturelles privées et les pratiques culturelles patrimoniales scolaires en invoquant deux arguments complémentaires: d'une part, il est nécessaire de développer un enseignement explicite des langages numériques multimodaux et de l'usage des supports numériques qui, loin d'être intuitifs, nécessitent une maitrise complexe pour laquelle l'apport des pairs peut également jouer tout son rôle (Penloup, 2017 ; Rispail, 2011). D'autre part, il est également nécessaire de relier deux sphères culturelles dont l'opposition renforce 
le désengagement et les difficultés scolaires des élèves les plus fragilisés dans leur rapport à la culture scolaire et académique.

C'est précisément en ce sens que s'interroge Fourtanier (2012), dans une réflexion centrée sur les pratiques littéraires :

Est-il souhaitable et possible et à quelles conditions d'intégrer la culture numérique

des jeunes aux cursus scolaires et aux dispositifs didactiques afin que les élèves

deviennent des lecteurs/producteurs de textes et d'images motivés et critiques?

(n. p.)

Selon elle, il est central, en contexte scolaire, pour limiter la "dislocation» qui empêche l'individu de "faire des liens, d'établir des relations, des ponts entre des objets de culture divers » et de s'appuyer sur les potentialités des environnements numériques pour favoriser les pratiques des élèves avec les œuvres littéraires. C'est dans une telle orientation que nous concevons notre dispositif expérimental.

\section{Cadre méthodologique}

\subsection{Conception du projet expérimental}

Notre démarche relève d'un projet de didactisation, c'est-à-dire d'une "opération consistant à transformer ou à exploiter un document langagier brut pour en faire un objet d'enseignement » (Cuq, 2003, p. 71) ; il ne s'agit pas de « scolariser » la fanfiction, d'en faire un objet scolaire, mais d'en proposer une adaptation qui vise à développer les compétences littéraires des élèves.

20 Il est question de transposer, d'adapter et non de naturaliser une pratique. En nous référant au modèle didactique du genre conçu par De Pietro et Schneuwly (2003), nous considérons que les genres enseignés ne forment en effet pas le "décalque " des genres sociaux, l'opération de transposition n'est pas "transparente» mais transformative, le critère central de cette transformation résidant dans son «efficacité pour atteindre les objectifs didactiques de transformation des modes de penser et de parler des élèves » (p. 49).

Nous avons ainsi sélectionné (Brunel, 2018a) certains éléments caractéristiques des fanfictions (le lien des écrits produits avec l'œuvre source; l'occasion de combler des blancs du texte), et certaines caractéristiques numériques des blogues de fanfiction (une même plateforme numérique pour lire, écrire et commenter ; un espace de forum, etc.). Puis, nous avons conçu des dispositifs relevant de pratiques fanfictionnelles, en relation avec la lecture d'une œuvre intégrale en classe (Brunel, 2018b). Dans ce processus, ont été écartés certains aspects des pratiques culturelles sociales de fanfiction pour préserver l'objectif d'enseignement :

- Du côté des objets enseignés, les œuvres supports ne sont pas choisies par les lecteurs, mais par l'enseignant dans le but de permettre aux élèves d'accéder à des œuvres classiques auxquelles ils n'auraient pas accès de manière autonome, telles que, en classe de $3^{\mathrm{e}}$ par exemple, Le Collier rouge de J.-C. Rufin, ou Le Meilleur des mondes de A. Huxley.

- Du côté des activités d'écriture, il s'agit d'orienter les écrits fanfictionnels ou commentatifs en fonction des compétences scripturales à enseigner au collège. Par exemple, en proposant d'insérer un nouvel épisode, c'est la compétence à produire un récit complet qui est exercée.

- Du côté des modalités pédagogiques, les regroupements en binômes et trinômes sont privilégiés, notamment pour favoriser les interactions entre pairs face à une tâche proposée 
sur écran (Baron \& Depover, 2019) tandis que, dans les pratiques sociales, la fanfiction s'écrit de manière individuelle. scolaire, les risques qui se présentent sont multiples :

- dénaturer la pratique sociale au risque de désengager les élèves percevant plus fortement le cadre scolaire du dispositif que sa parenté avec des pratiques sociales attractives ;

- verser dans une dérive occupationnelle proposant des pratiques qui séduisent les élèves mais ne favorisent pas le développement de leurs compétences ;

- proposer un cadre didactique et pédagogique si éloigné du cadre conventionnel de la classe qu'il insécurise les élèves provoquant leur refus à s'engager dans les activités.

C'est principalement à l'aune du premier de ces risques que nous analyserons les résultats de notre expérimentation.

\subsection{Contexte de l'expérimentation}

L'expérimentation d'adaptation de la fanfiction au collège a concerné une classe de $3^{\mathrm{e}}$ d'un collège varois, pendant une année scolaire. Le travail de la classe a été analysé et les résultats des évaluations de chaque séquence d'étude d'œuvres a été comparé - à partir d'évaluations communes - aux résultats d'une autre classe de $3^{\mathrm{e}}$ menant les mêmes études avec le même professeur sans employer la fanfiction. Trois œuvres ont été étudiées (une par trimestre), et trois fanfictions ont donc été proposées.

Un questionnaire de début d'expérimentation a mis en évidence le fait que les vingtdeux élèves de la classe avaient des pratiques culturelles personnelles régulières sur écran (jeux, réseaux sociaux, vidéos sur YouTube ou séries, mais également recherches documentaires). Parmi eux, seuls trois connaissaient les fanfictions, mais ils n'en avaient jamais produit. Enfin, lorsqu'on leur demande sur quel support ils préfèrent écrire, quatre d'entre eux indiquent préférer écrire sur papier, tandis que les dix-huit autres précisent qu'ils préfèrent écrire sur écran. Dans un cadre plus scolaire, précisons que ces élèves ne possèdent pas une habitude, en classe de français, d'usage du matériel numérique, même si dans d'autres disciplines, ils viennent travailler dans la salle informatique qui est utilisée au cours de l'expérimentation fanfiction. À deux exceptions près, tous possèdent chez eux un ordinateur ou une tablette leur permettant de poursuivre à la maison les travaux de fanfiction engagés en classe. Les deux élèves qui n'en sont pas dotés se voient proposer des plages de mise à disposition d'ordinateurs au centre de documentation et d'information (CDI) 3 .

\subsection{Présentation de la ressource numérique et du scénario didactique}

La plateforme numérique institutionnelle Moodle constitue le support de la création d'un espace, nommé "fanfiction + nom de l'œuvre lue », pour chaque œuvre étudiée. Elle se structure en espaces de lecture de type forum (fig. 1) et en espaces d'écriture fanfictionnelle (fig. 2) associés à des pages recueillant les commentaires des lecteurs 4 . Elle vise à se rapprocher, dans son ergonomie et sa structuration, de sites de blogues fanfictionnels. 
Figure 1. - Présentation du forum de lecture de la fanfiction « La Ferme des animaux».

\section{Fanfiction La Ferme des animaux}

\section{Forum informel}

Ici, tu peux déposer tes impressions de lecture librement, poser des questions, aider tes camarades dans leur lecture...

\section{Afouter une discussion}

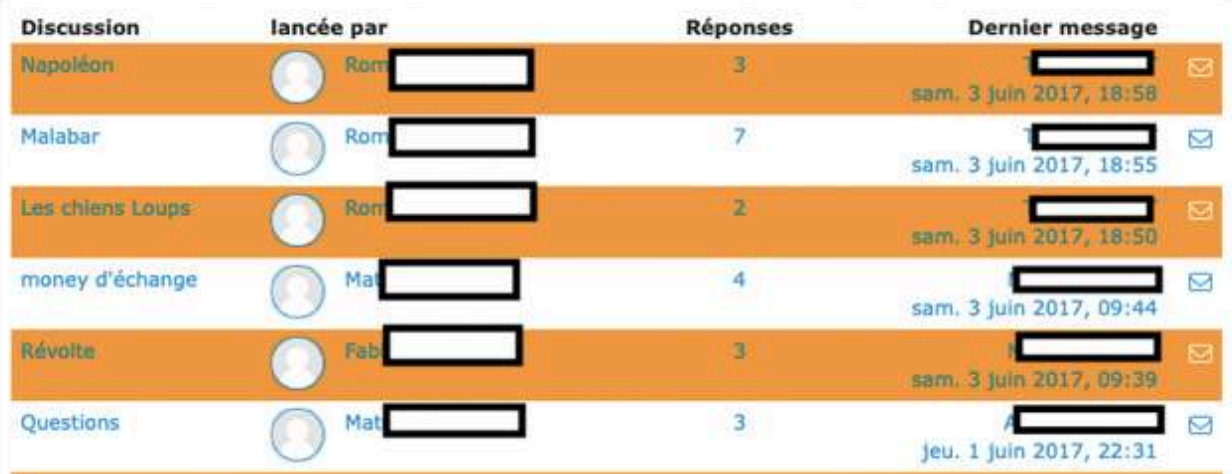

Figure 2. - Extrait des commentaires produits par les élèves du groupe 3 pour la fanfiction "La Ferme des animaux ».

\section{version 1}

Ajouter commentaire

\section{oar $5:$ mardi 23 mai 2017, 15:28}

Dans notre texte nous avons choisit l'utilisation de la violence car nous avons estimer que la maniere pacifiste ne marcher pas pour arreter napoleon de monter tout le monde contre jhons. Nous avons ete du coter de boule de neige mais nous avons quand meme fait l'usage de la violence.

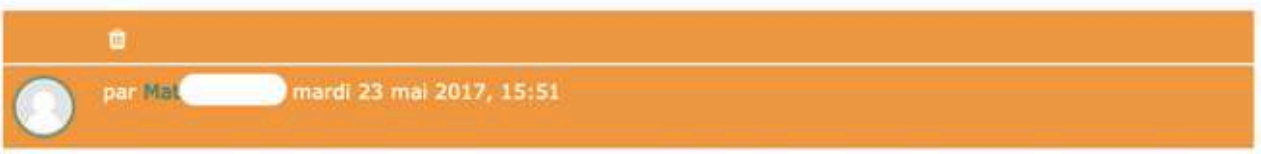

Nous trouvons très interréssant de faire revenir car il était l'ancien dirigeant de I ferme. Cepandant nous trouvons votre histoire un peu courte pour que la ferme redevienne prospère aussi rapidement.

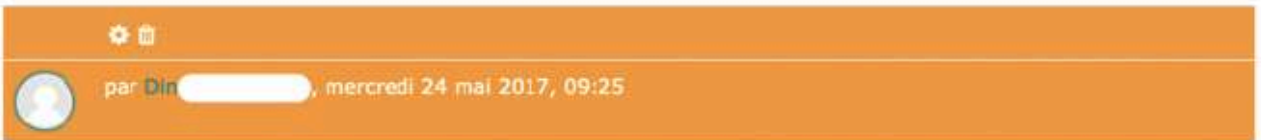

Je trouve votre texte très intéressant, l'idée de faire revenir l'ancien propriétaire de la ferme est excellente. Dina-Perrine

La séquence d'apprentissage servant de canevas à la mise en œuvre de l'expérimentation associe des supports et propositions d'activités d'écriture et de lecture proches des pratiques fanfictionnelles tout en respectant le cadre contraint des programmes. La plateforme fanfiction est notamment employée en lecture : les forums offrent les supports d'appuis de débats interprétatifs, cercles de lecture ou parcours de lecture. En écriture, l'essentiel des activités consiste en l'écriture de textes fanfictionnels, à partir des propositions de l'enseignant, puis en lecture et commentaires des productions des camarades et enfin, en des activités de réflexivité 
et de réécritures individuelles ou collectives. Le lien entre espace privé et espace scolaire, entre l'individu et le groupe se trouve facilité par l'accès, quel que soit le moment ou le lieu, à la plateforme de l'établissement. Enfin, chaque séance commence par un rituel de "retour sur les forums ", à travers lequel l'enseignant aménage un espace de réaction subjective (Massol \& Rannou, 2017) et de discussion (Sauvaire, 2013), qui permet de mettre en relation la lecture personnelle des élèves, les échanges du forum et les activités de classe. Voici un exemple de séquence adaptée du canevas expérimental, qui a été menée au cours du premier trimestre sur Le Collier rouge ${ }^{5}$.

Tableau 1. - Présentation de la séquence Le Collier rouge avec codage des verbatims.

\begin{tabular}{|c|c|c|}
\hline $\begin{array}{l}\text { Séances } \\
\text { filmées }\end{array}$ & Contenu de la séance & Codage \\
\hline $\begin{array}{l}\text { Séquence } 1 \\
\text { Séance } 1\end{array}$ & $\begin{array}{l}\text { Découverte de l'œuvre Le Collier rouge et de la plateforme de fanfiction et } \\
\text { des forums }\end{array}$ & V1 \\
\hline $\begin{array}{l}\text { Séquence } 1 \\
\text { séance } 2\end{array}$ & Étude de l'incipit (lecture analytique) & V2 \\
\hline \multirow[t]{2}{*}{$\begin{array}{l}\text { Séquence } 1 \\
\text { séance } 4\end{array}$} & Écriture $-1^{\mathrm{er}}$ jet de la proposition « modifier un épisode » & V3 \\
\hline & Écriture - Retour sur les versions et les commentaires & V4 \\
\hline $\begin{array}{l}\text { Séquence } 1 \\
\text { séance } 5\end{array}$ & $\begin{array}{l}\text { Lecture transversale sur le rôle du chien à partir d'un fil de discussion du } \\
\text { forum }\end{array}$ & V5 \\
\hline \multirow[t]{2}{*}{$\begin{array}{l}\text { Séquence } 1 \\
\text { séance } 6\end{array}$} & Écriture - Comprendre la fonction des commentaires et en produire & V6 \\
\hline & (suite de la séance) & V7 \\
\hline $\begin{array}{l}\text { Séquence } 1 \\
\text { séance } 9\end{array}$ & Parcours de lecture (travail de groupe) : les personnages secondaires & V8 \\
\hline $\begin{array}{l}\text { Séquence } 1 \\
\text { séance } 10\end{array}$ & Écriture - $1^{\mathrm{er}}$ jet de la proposition : « Un personnage raconte» & V9 \\
\hline \multirow[t]{2}{*}{$\begin{array}{l}\text { Séquence } 1 \\
\text { séance } 11\end{array}$} & Écriture - Écriture de la fanfiction en groupe d'écriture en classe & V10 \\
\hline & (suite de la séance) & V11 \\
\hline $\begin{array}{l}\text { Séquence } 1 \\
\text { séance } 12\end{array}$ & Bilan synthétique & V12 \\
\hline
\end{tabular}

Les données que nous mobilisons pour notre étude sont les suivantes :

- les transcriptions des séances de fanfiction filmées ;

- les productions des élèves dans le blogue numérique fanfictionnel ; 
- les résultats des évaluations des bilans de fin de séquence, en lecture et écriture, (équivalentes à celles données dans la classe ne bénéficiant pas du dispositif).

\section{Analyses} contexte scolaire, telle que nous l'avons mise en œuvre, peut durablement rester attractive et occasionner une évolution du rapport à l'écrit des lecteurs/scripteurs. Pour répondre à cette question, nous nous appuyons sur trois indicateurs issus du traitement de nos données. Tout d'abord, nous considérons que les éléments chiffrés obtenus par le système informatique constituent des données indirectes pertinentes pour mesurer l'engagement des élèves dans la pratique fanfictionnelle. En effet, les chiffres de fréquentation de la plateforme tout au long de l'année permettent d'identifier le nombre d'élèves qui interviennent sur la plateforme, la durée de leur intervention, leur nature et le moment de celle-ci. D'autre part, nous suivons Marin et Crinon (2017) qui considèrent que la longueur du texte constitue un critère de mesure d'un niveau de performance d'écriture même si elle ne peut constituer un élément suffisant pour évaluer un écrit. Nous nous appuierons sur cet indicateur pour mesurer l'investissement des élèves, celui-ci étant lui-même un signe de leur rapport à l'écrit ; enfin, nous retenons, au sein des verbatims que nous sélectionnons, les passages où les élèves s'interrogent et commentent la situation de communication de blogue fanfictionnel : ces extraits nous paraissent constituer des indicateurs de leur prise de conscience des fonctions de l'écrit.

\subsection{Le développement de la fréquentation de la plateforme}

L'espace Moodle mis en place permet de garder mémoire de l'activité des élèves : de la sorte, il est possible de connaitre le nombre d'utilisations de la plateforme fictionnelle tout au long de la mise en place de l'expérimentation. En outre, l'outil de statistiques permet de distinguer les activités de consultation - c'est-à-dire la lecture des différents forums - des activités de production de posts sur ces mêmes forums. Ainsi, le tableau suivant permet de suivre comment l'utilisation des forums des 22 élèves de la classe évolue au cours de l'étude des trois œuvres intégrales abordées dans l'année.

Tableau 2. - Synthèse des activités sur les forums de la plateforme fanfiction.

\begin{tabular}{|l|l|l|l|}
\hline Activité de la classe sur les forums & $\begin{array}{l}\text { Euvre intégrale 1 } \\
\text { septembre-octobre }\end{array}$ & $\begin{array}{l}\text { CEuvre intégrale 2 } \\
\text { janvier-février }\end{array}$ & $\begin{array}{l}\text { Euvre intégrale 3 } \\
\text { avril-mai }\end{array}$ \\
\hline Total des consultations de pages & 787 & 839 & 1084 \\
\hline Total des messages postés & 103 & 102 & 146 \\
\hline
\end{tabular}

Les données du tableau révèlent que l'activité des élèves se développe au cours de l'année, aussi bien en consultation qu'en production de message. Ainsi, l'attractivité de la plateforme fanfictionnelle ne se limite pas à l'effet de séduction qu'aurait pu 
occasionner la première séquence, mais perdure et se renforce. Les élèves investissent ainsi de plus en plus ces espaces qu'ils utilisent en majorité hors du temps scolaire.

Ce que le tableau permet particulièrement de montrer, c'est le développement d'une «habitude ", l'adoption d'un usage : il semble que les élèves qui ont une habitude de consultation de réseaux privés ont en quelque sorte adapté ces usages à la consultation de la plateforme scolaire de fanfiction. Nous considérons que la proximité du format et du mode de fonctionnement avec ceux d'une plateforme informelle ou d'un réseau social a favorisé une telle adoption et a permis de s'appuyer sur leurs compétences déjà-là (Cordier, 2015).

Cependant nous notons un écart très sensible entre les activités de consultation et celles de post de messages: même si celui-ci est logique, car toute consultation ne donne pas lieu à écriture, il montre que souvent, l'utilisation des forums ne se réalise qu'en réception. Le forum constitue donc moins une occasion de production personnelle qu'un moyen de s'informer sur les avis des pairs. Pourtant, l'écart entre les deux usages se réduit en cours d'année : certains élèves se familiarisent peu à peu avec la pratique du forum et commencent à y contribuer plus tardivement. Comme tout apprentissage d'un genre écrit nouveau, le genre "forum» nécessite une forme d'acculturation qui se réalise d'abord à travers la lecture et exige une certaine durée.

Ajoutons que, même dans le cas où les élèves en restent à une pratique consultative, les chiffres obtenus attestent d'un suivi régulier, et même de plus en plus régulier : nous identifions ainsi que les consultations, et dans une moindre mesure, les interventions sur le forum accompagnent la lecture et ont peut-être pu permettre de limiter le risque de décrochage. Dans ce cadre, il nous apparait que le rapprochement entre pratiques privées et pratiques scolaires ici opéré a pu particulièrement profiter aux élèves qui sont les plus fragilisés lorsque ce rapport se construit dans un antagonisme (Cordier, 2015). Dès lors, il a sans doute permis de rapprocher les œuvres littéraires lues des objets culturels informels des élèves, en intégrant ces objets plus classiques au sein de pratiques qui leur sont plus familières, comme le propose Fourtanier (2012).

\subsection{Le développement du gout pour l'écriture}

35 Dans notre dispositif expérimental, les élèves de la classe test ne réalisent, au cours de la séquence, que des activités d'écriture de type fanfiction, sur écran. En revanche, l'évaluation, qui est commune aux élèves de la classe test et à ceux de la classe témoin, est pratiquée selon un format de type brevet et consiste en une activité de suite de texte, accompagnée de consignes. Si les deux productions (fanfiction et écriture d'invention certificative) sont finalement proches dans les compétences qu'elles mobilisent, elles correspondent à des consignes, des supports de production et un contexte d'écriture bien différents. Parmi l'ensemble des éléments permettant d'évaluer une production, nous privilégions ici le critère de la longueur ${ }^{6}$, mesurable quantitativement. En effet, si celui-ci ne permet pas d'évaluer la qualité de l'écrit, il permet d'effectuer une mesure révélant une forme d'engagement dans l'activité (Marin \& Crinon, 2017). Nous nous proposons ainsi de mesurer l'évolution de la longueur des écrits des élèves de la classe test, dans les différentes productions.

Tout d'abord, le tableau suivant permet de mesurer l'évolution générale de la longueur des textes produits dans les différents groupes ${ }^{7}$. 
Tableau 3. - Synthèse de la longueur des productions des groupes de la classe (en nombre de lignes).

\begin{tabular}{|c|c|c|c|c|c|c|}
\hline & $\begin{array}{l}\text { Séquence fanf } \\
\text { « Le Collier ro }\end{array}$ & $\begin{array}{l}\text { fiction } 1 \\
\text { uge » }\end{array}$ & $\begin{array}{l}\text { Séquence fant } \\
\text { « Le Meilleur }\end{array}$ & $\begin{array}{l}\text { fiction } 2 \\
\text { des mondes » }\end{array}$ & $\begin{array}{l}\text { Séquence fanf } \\
\text { « La Ferme de }\end{array}$ & $\begin{array}{l}\text { iction } 3 \\
\text { s animaux » }\end{array}$ \\
\hline Groupes (1) & Production A & Production B & Production A & Production B & Production A & Production B \\
\hline G1 & $23(2)$ & 15 & 17 & 36 & 22 & 17 \\
\hline G2 & 11 & 51 & 21 & 14 & 21 & 20 \\
\hline G3 & 13 & 30 & 21 & 14 & 21 & 20 \\
\hline G4 & 9 & 15 & 21 & 11 & 11 & 27 \\
\hline G5 & & 16 & 29 & 23 & 14 & 41 \\
\hline G6 & & 34 & 14 & 11 & 17 & 2 \\
\hline G7 & & 4 & 22 & 6 & 16 & 23 \\
\hline G8 & & & 16 & 14 & 11 & 23 \\
\hline G9 & & & 28 & 14 & 14 & 17 \\
\hline $\begin{array}{l}\text { G10 } \\
\text { (1 élève) }\end{array}$ & & & & 13 & 17 & 13 \\
\hline $\begin{array}{l}\text { G11 } \\
\text { (1 élève) }\end{array}$ & & & & 24 & 25 & \\
\hline $\begin{array}{l}\text { Total de } \\
\text { lignes } \\
\text { écrites par } \\
\text { la classe }\end{array}$ & 56 & 165 & 179 & 179 & 180 & 192 \\
\hline $\begin{array}{l}\text { Moyenne } \\
\text { de lignes } \\
\text { écrites par } \\
\text { groupe }\end{array}$ & 14 & 23 & 21 & 17 & 17 & 20 \\
\hline
\end{tabular}

Les chiffres totaux révèlent un allongement moyen des écrits. Celui-ci peut être repéré dans le nombre total de lignes rédigées par la classe - de 56 à 192 lignes -, mais également en pondérant ce nombre en fonction du nombre de groupes : dans ce cas, l'allongement moyen évolue de 14 à 20 lignes. Même si le phénomène n'est pas parfaitement linéaire, tous les textes qui suivent la première production manifestent une progression. L'analyse du tableau nous permet également de repérer que le nombre de groupes évolue. En effet, l'enseignant propose tout au long de l'année aux élèves de se regrouper librement, en suggérant la composition de groupes de 2 à 4 élèves. Mais peu à peu, les élèves eux-mêmes réclament de scinder les groupes, voire d'écrire seuls. Cette évolution conduit chacun à produire davantage et de manière de plus en plus 
personnelle. La composition de la classe évolue ainsi de 4 à 10 groupes dès le milieu de l'année. Nous considérons que cette évolution constitue également un indicateur de l'investissement des élèves dans l'activité et d'un rapport à l'écrit qui se développe positivement et de manière de plus en plus personnelle (Tauveron \& Sève, 2005).

Enfin, la mesure de la longueur des productions des élèves lors des évaluations en production écrite de fin de séquence apporte un résultat convergent avec celle des textes produits en fanfiction : tandis qu'en début d'année, les élèves - de la classe test comme ceux de la classe témoin - s'efforcent, au moment des évaluations de type brevet, de respecter les normes de longueur indiquées dans les consignes, on voit apparaitre ensuite, et ce uniquement dans la classe testée, une tendance à dépasser la norme attendue. Ceci est identifiable dans deux copies lors du second test évaluatif et dans huit $\operatorname{copies}^{8}$ (soit plus d'un tiers de la classe), lors du troisième test, certaines productions comportant jusqu'à six pages. Ainsi, comme les processus d'écriture à l'œuvre dans les productions réalisées dans le cadre de la fanfiction restent proches de ceux impliqués dans les sujets d'invention proposés dans les évaluations (Cauterman, 2003), les élèves semblent transférer les compétences qu'ils ont mobilisées dans les premières vers les secondes.

L'ensemble de ces indicateurs nous permet de considérer que le dispositif expérimental a pu stimuler durablement la productivité des élèves, ce qui nous parait constituer un signe tangible de l'évolution positive de leur rapport à l'écrit.

\subsection{La prise de conscience du sens et des fonctions de l'écrit}

Les verbatims révèlent que les productions fanfictionnelles se chargent d'un enjeu réel pour les élèves : ils se sentent valorisés d'être publiés et soucieux de la réception de leurs textes. À titre d'exemple, nous présentons un extrait du verbatim d'une séance située au début de l'expérimentation, dans laquelle le professeur aborde le fait que la production des textes sur la plateforme constitue une publication :

P. - On va aller voir vos travaux.

E26. - Non madame, s'il vous plait, vous êtes obligée de les lire ?[...]

P. - Non non mais n'ayez pas peur. Tout le monde a accès à ce que vous avez écrit. Au contraire, c'est pour vous féliciter. Donc, le groupe 1 et le groupe 2, je vous félicite, puisque dans le groupe 1 comme dans le groupe 2 , on trouve une version 1 . Ensuite, moi, je leur ai déposé un commentaire [...]

Mae. - Madame on vous répond au commentaire que vous avez mis ou pas?

P. - Tu peux faire ce que tu veux, si tu veux me répondre, tu me réponds. Si t'as envie de répondre, tu réponds.

Lilly. - Ça nous a fait plaisir c'est un plutôt beau commentaire !

Mae. - Ouais, merci madame.

P. - Ça vous a fait plaisir mon commentaire?

Mae. - Oui !

P. - Bon, ben, c'est formidable !

(Extrait de la séance 2)

Dans cet extrait, l'élève E26 comprend, lorsque le professeur le lui explicite, que son texte, sur la plateforme, peut être lu par toute la classe, et comme on le perçoit à son inquiétude, sa perception de l'écrit évolue. C'est la même prise de conscience qui provoque chez Lilly et Mae, au contraire, une réaction d'enthousiasme, comme le révèlent leurs remerciements et leur souhait de prolonger l'échange avec leur professeur-lecteur. Ces différentes interventions constituent un premier signe de 
compréhension de la dimension sociale de l'écrit fanfictionnel numérique. D'autres échanges, portant sur la situation de communication dans laquelle ils s'inscrivent, mettent en évidence, au cours de l'année, le fait que les élèves cernent mieux les enjeux d'une publication numérique.

\section{Discussion et conclusion}

Face aux trois risques liés à l'adaptation de pratiques numériques adolescentes en contexte scolaire que nous avons précédemment évoqués, notre questionnement se centrait sur celui du désengagement des élèves. Nous avons ainsi cherché à identifier si l'adaptation en contexte scolaire d'une plateforme de fanfiction pouvait susciter un intérêt durable, facteur d'engagement des élèves et favorisait un rapport à l'écrit plus positif. Plusieurs indicateurs nous ont permis de mesurer le fait que le dispositif n'a pas occasionné d'effet déceptif sur la durée. De véritables habitudes de consultations de la plateforme sont constatées ce qui rapproche l'usage de cette ressource scolaire des pratiques privées que les élèves peuvent avoir des réseaux sociaux. La proximité de l'ergonomie et du fonctionnement en "communauté » de l'interface fanfiction a pu favoriser la sollicitation d'usages et de compétences informelles et rapprocher les activités scolaires des pratiques culturelles privées. Nos analyses semblent aussi attester du fait que le rapport à l'écrit des élèves a évolué de manière positive comme le montrent l'allongement de leur écrit et certaines de leurs interventions en classe - même si nous ne négligeons pas la multiplicité des dimensions intervenant dans ce rapport (Barré-De Miniac, 2000). Nous considérons qu'une forme de profit mutuel s'exerce entre pratique d'écriture numérique sociale et développement de compétences scolaires : c'est à travers l'usage de la plateforme numérique de fanfiction interne à la classe que les élèves parviennent à identifier certaines fonctions fondamentales de l'écrit, et en retour, l'activité de production sur les forums en contexte scolaire leur permet de développer des compétences sur la littératie numérique (prise de conscience du caractère public de leurs écrits, appréhension de formats spécifiques tels que les billets ou les commentaires).

Il n'est pas question ici d'imputer ces résultats positifs au seul caractère numérique du dispositif: la stimulation de la subjectivité et de la créativité dans les propositions d'écriture fanfictionnelle ou encore la posture plus collaborative qu'évaluative de l'enseignante, constituent également des conditions susceptibles d'avoir contribué à ces résultats. C'est bien plutôt l'ensemble du dispositif, mobilisant plusieurs facteurs et non « la simple présence de technologies numériques qui change les choses » (Baron \& Depover, 2019, p. 84).

Si notre étude ne visait pas à évaluer les autres risques occasionnés par l'adaptation, nos analyses permettent cependant de percevoir des manifestations de malaise ou de résistance de certains élèves devant le cadre didactique et pédagogique inhabituel qui leur a été proposé. Ainsi, nous avons identifié à travers l'écart des mesures entre les consultations et les productions le fait que certains élèves n'intervenaient pas spontanément ${ }^{9}$ sur les forums. De même, dans l'extrait présenté en exemple, un élève se montre inquiet en réalisant que son écrit est visible par tous. Ces limites à l'attractivité du dispositif interrogent: ces attitudes relèvent-elles davantage d'une inquiétude à agir en étant visible par ses pairs (et donc d'une résistance liée à la préservation de la face) que d'une réticence à entrer dans une nouvelle activité (Hélou 
\& Lantheaume, 2008)? Peut-on les interpréter comme des craintes à s'engager personnellement dans le dispositif didactique proposé, quand les formats scolaires semblent rendre davantage possible une posture d'extériorité ? Peut-on au contraire y voir une forme de méfiance pour une ressource qui malgré tout, reste scolaire?

L'étude d'entretiens post-expérimentation aurait pu sans doute permettre de mieux percevoir les causes des résistances de certains élèves et de mieux les appréhender, dans une phase ultérieure du projet. Il s'agit là d'une limite de notre dispositif de recherche.

Il reste que l'adaptation de fanfiction expérimentée a suscité l'intérêt durable d'une majorité d'élèves et a assoupli l'opposition entre des pratiques rattachées à la culture scolaire et celles rattachées à la culture informelle. Les élèves se sont investis dans la lecture d'œuvres littéraires classiques à travers un dispositif numérique qui a contribué à créer des habitudes de lecture, qui a motivé leur engagement et leur a permis de mieux comprendre les enjeux de l'écrit, notamment en contexte numérique (Cordier, 2015). Dès lors, il serait pertinent de suivre Beckett et Hager (2002) qui repoussent l'idée d'une démarcation stricte entre les différentes sphères d'apprentissage. En ce sens, l'introduction des littératies numériques en contexte scolaire pourrait soutenir de nouvelles conceptions de l'apprentissage qui «brouillent cette démarcation » et avancent plutôt «l'idée d'un continuum de l'informel au scolaire»(ibid., p. 154) - et réciproquement.

\section{BIBLIOGRAPHIE}

BARNABÉ, Fanny. (2014). Ludicisation des pratiques d'écriture sur Internet : une étude des fanfictions comme dispositifs jouables. Sciences du jeu, 2. <https://doi.org/10.4000/sdj.310>. BARON, Georges Louis \& DEPOVER, Christian. (2019). Les effets du numérique sur l'éducation. Presses universitaires du Septentrion.

BARRÉ-DE MINIAC, Christine. (2000). Le rapport à l'écriture, aspects théoriques et didactiques. Presses universitaires du Septentrion.

BeCKetT, David \& Hager, Paul. (2002). Life, Work, and Learning: Practice in Postmodernity. Routledge.

BRUNEL, Magali. (2018a). Les écrits de fanfiction dans la classe. Le français aujourd'hui, Écriture numérique : la conversion du littéraire ?, 200, 31-42. <www.cairn.info/revue-le-francais-aujourdhui-2018-1-page-31.htm>.

BRUNEL, Magali. (2018b). La fanfiction, une ressource pour lire l'œuvre intégrale. Recherches : revue de didactique et de pédagogie du français, Usages du numérique, 69, 51-70.

CAUterman, Marie-Michèle. (2003). Les sujets d'écriture d'invention au collège et au lycée. Recherches : revue de didactique et de pédagogie du français, Écriture d'invention, 39, 81-96.

CENTRE NATIONAL DE LA LECTURE. (2016). Les jeunes et la lecture. <https://centrenationaldulivre.fr/ donnees-cles/les-jeunes-et-la-lecture>. 
COMMISSION EUROPÉENNE. (2012). Proposition de Recommandation du Conseil relative à la validation de l'apprentissage non formel et informel.

CORDIER, Anne. (2015). Grandir connectés, les adolescents et la recherche d'information. C\&F Éditions.

CuQ, Jean-Pierre (dir.). (2003). Dictionnaire de didactique du français. Clé International.

De PIETRo, Jean-François \& SCHNeuwly, Bernard. (2003). Le modèle didactique du genre : un concept de l'ingénierie didactique. Les cahiers Théodile, 3, 27-52.

DABÈNE, Michel. (1991). Un modèle didactique de la compétence scripturale. Repères, 4, 9-22.

DiAz, Ana. (2015). Le débat interprétatif dans l'enseignement du français. Peter Lang.

DoueIHI, Milad. (2011). Pour un humanisme numérique. Seuil.

FrançoIs, Sébastien. (2009). Fanf(r)ictions : tensions identitaires et relationnelles chez les auteurs de récits de fans. Réseaux, 153, 157-189. <https://doi.org/10.3917/res.153.0157>.

FOURTANIER, Marie-José. (2012). Petits Poucets, petites Poucettes dans la forêt d'Internet : les élèves lecteurs à l'heure du numérique. Communication présentée au colloque « Les temps et les lieux de la lecture », Sherbrooke, Québec, Canada.

HÉLOU, Christophe \& LANTHEAume, Françoise. (2008). Les difficultés au travail des enseignants. Recherche et formation, 57, 65-78.

LACELLE, Nathalie, Boutin, Jean-François \& LeBrun, Monique. (2017). La littératie médiatique multimodale appliquée en contexte numérique. Presses universitaires du Québec.

LAFONT-TERRANOVA, Jacqueline. (2009). Se construire à l'école comme sujet écrivant, l'apport des ateliers d'écriture. Presses universitaires de Namur.

MARIN, Brigitte \& CRINON, Jacques. (2017). Le déjà-là et le presque-là. Pratiques, 173-174. <https:// doi.org/10.4000/pratiques.3364>.

MASSOL, Jean-François \& RANNOU, Nathalie. (2017). Le sujet lecteur-scripteur de l'école à l'université. Presses universitaires de Grenoble.

MAZAuric, Catherine, Fourtanier, Marie-Josée \& LANGLADE, Gérard (dir.). (2011). Le texte du lecteur. Peter Lang.

MigUET, Mathilde. (2014). Livres numériques : stratégies des lecteurs dans leurs pratiques. Études de communication, 43, 57-74. <https://doi.org/10.4000/edc.5991>.

MinistÈRE DE L'ÉDUCATION NATIONALE. (2015). Programmes d'enseignement du cycle des apprentissages fondamentaux (cycle 2), du cycle de consolidation (cycle 3) et du cycle des approfondissements (cycle 4). Bulletin officiel spécial $\mathrm{n}^{\circ} 11 \mathrm{du} 26$ novembre 2015. <www.education.gouv.fr/pid285/ bulletin_officiel.html?pid_bo=33400>.

Penloup, Marie-Claude. (2017). Didactique de l'écriture : le déjà-là des pratiques d'écriture numérique. Le français aujourd'hui, 196, 57-70.

RENARD, Fanny. (2011). Les lycéens et la lecture. Entre habitudes et sollicitations. Presses universitaires de Rennes.

RISPAIL, Marielle. (2011). Littéracie : une notion entre didactique et sociolinguistique. Enjeux sociaux et scientifiques. FJ forumlecture.ch, 2011(1). <www.leseforum.ch/fr/archiv.cfm? issue $=1 \&$ year $=2011>$. 
POYET, Françoise. (2011). Culture scolaire et culture numérique en tension. Dans F. Poyet \&

C. Develotte (dir.), L'éducation à l'heure du numérique (p. 29-46). ENS-INRP.

SAUVAIRE, Marion. (2013). Diversité des lectures littéraires. Comment former des sujets lecteurs divers?

(Thèse de doctorat, Université Toulouse 2-Le Mirail et Université Laval). <http://hdl.handle.net/

20.500.11794/24045>.

TAUVERON, Catherine \& SÈVE, Pierre. (2005). Vers une écriture littéraire, ou comment construire une posture d'auteur à l'école: de la GS au CM2. Hatier.

\section{NOTES}

1. <www.fanfic-fr.net/fanfics/Livres-Romans/L/Le-Hobbit.html?p=2>.

2. Les propositions d'écriture fanfictionnelles sont souvent proches des formes traditionnelles des sujets d'écriture; dès lors, il est nécessaire que les enseignants écartent « des sujets de rédaction qui seraient trop précis et associés à des consignes normatives » (Brunel, 2018b, p. 62).

3. L'expérimentation a été menée lors de l'année scolaire 2016-2017. L'année suivante, les élèves ont été dotés de tablettes.

4. Pour la présentation de la plateforme, voir Brunel (2018a et 2018b).

5. Toutes les séances de la séquence ont été filmées et transcrites sous forme de verbatim.

6. Tout comme dans les écrits lus sur les plateformes de fanfictions sur Internet, les différentes propositions d'écriture relèvent d'écrits fictionnels dont les normes ne présentent pas de limites de longueur attendues et que les scripteurs peuvent ainsi investir librement.

7. Les groupes ne sont pas toujours composés de la même manière. Aussi, il n'est pas possible de suivre l'évolution des groupes en se limitant à suivre les numéros de groupes de la plateforme.

8. Concernant les autres élèves de la classe, précisons que tous respectent les normes de longueur attendues.

9. Rappelons cependant qu'ils interviennent également sur ces forums en classe et que l'écart entre les deux mesures tend à se réduire au cours de l'année.

\section{RÉSUMÉS}

Notre étude consiste dans l'analyse d'un dispositif expérimental d'adaptation scolaire de pratiques de fanfiction dans le cadre de l'enseignement du français au collège. Elle repose sur le constat que la pratique littératique adolescente de la fanfiction favorise le développement de compétences littéraires et numériques et qu'elle présente des caractéristiques communes avec les pratiques d'écriture littéraires et même scolaires traditionnelles. En ce sens, elle peut présenter un potentiel didactique pertinent. Cependant rapprocher les pratiques informelles du cadre scolaire présente le risque que celles-ci perdent leur attractivité et ne permettent plus de déjouer un rapport négatif à l'écrit. Nous analyserons si l'expérimentation fanfiction, menée sur la durée d'une année scolaire, a pu permettre de stimuler durablement l'engagement des élèves et de faire évoluer positivement leur rapport à l'écrit.

Our study consists in the analysis of an experimental academic adaptation of fanfiction practices in the context of French teaching in middle schools. It is based on the observation that the 
adolescent practice of fanfiction fosters the development of literary and numerical skills and that it presents common characteristics with traditional literary and even academic writing practices. In this sense, it may have relevant didactic potential. However, bringing informal practices closer to the school setting is a risk as they might then lose their appeal and no longer make it possible to thwart negative relationships with the written word. We will analyze whether or not the fanfiction experiment, carried out over the course of a school year, has been able to stimulate the students' commitment and positively change their relationship to the written word.

\section{INDEX}

Keywords : digital literacy, writing, teaching, literature, motivation

Mots-clés : littératie numérique, écriture, enseignement, littérature, motivation

\section{AUTEUR}

\section{MAGALI BRUNEL}

Université Côte d'Azur, Laboratoire d'innovation et numérique pour l'éducation 